\title{
Generalizing Multiple Examples in Explanation Based Learning
}

\author{
Gunther Sablon \\ Luc De Raedt \\ Maurice Bruynooghe \\ Departement of Computer Science, K.U.Leuven \\ Celestijnenlaan 200A, B-3030 Heverlee, Belgium \\ Phone : $+32 / 16 / 200656$ \\ Email ...mcvax!prlb2!kulcs!gunther, \\ gunther@kulcs.uucp \\ Telex : 23674 kuleuv b Telefax : $+32 / 16 / 205308$
}

\begin{abstract}
Presenting multiple examples to an Explanation Based Learning system may lead to a lot of quite similar rules. This has a negative effect on the overall problem solving performance. The problem can be alleviated by combining several mules into one. We present a method to generalize rules by locating common parts and differences in order to obtain a more useful set of rules.
\end{abstract}

\section{Introduction.}

In most Explanation Based Learning systems (cf. [Mitchell 86], [De Jong 86], [Hirsch 87], [KedarCabelli 87]) learning from multiple examples leads to a lot of rules, one for each example. In recent work (cf. [Minton 87], [Keller 87], [Minton 88]) it is recognized that not all leamed concept descriptions have the same level of utility. Because most EBL systems operationalize rules instead of concepts (cf. [Keller 87]), some concept descriptions may contain very specific and redundant rules. One way to achieve concept operationalization is to derive more general rules and operationalize the generalized subconcepts where possible. In this way, one tries to find an equilibrium (possibly temporarily) in the generality/operationality trade-off (cf.[Segre 87]) to improve the operationality of the whole concept. In the rest of the paper we assume that operationality of a concept description takes into account (as in [Keller 87]) : usability by the performance system (leamed rules must be evaluable) and utility to the performance system (learned rules must improve the systems performance).

We define a concept as a set of objects from a universe, determined by a concept definition. To describe concept definitions we use the Horn Clause subset of first order logic (cf. [Kowalski 79], [Genesereth 87] for more details on Horn Clause Logic and its terminology). A concept definition is a set of Horn clauses that expresses the necessary and sufficient conditions to belong to the concept.

In the second section we briefly review classical EBL and in the third one we discuss its drawbacks when confronted with multiple examples. In the fourth section we present a solution which generalizes the rules. It will be demonstrated on an example. After a discussion we finally discuss related work.

*) Supported by the Belgian National Fund for Scientific Research. 


\section{Explanation Based Learning.}

The four inputs of a classical Explanation Based Learning system (cf. [Mitchell 86],[De Jong 86]) are:

- a concept definition of the concept to be operationalized

- a training example

- a domain theory : a set of rules, describing why the training example is a positive example

- an operationality criterion.

By means of these four inputs one tries to determine an operational generalization of the training example, which forms a sufficient condition to belong to the concept. General knowledge is transformed into more operational knowledge.

EBL can be considered as lemma generation (cf. [Kowalski 79]) controlled by an example. In the earlier EBL systems only the usability aspect of the operationality criterion was taken into account. When a clause $A \leftarrow B_{1}, \ldots, B_{m}$ is used to solve the example, $B_{i}$ is a call to a non-usable concept and, in the example, a clause $P \leftarrow C_{1}, \ldots, C_{k}$ is used to solve the instance of $B_{i}$, then resolution is applied on both clauses and the resolvent $\left(A \leftarrow B_{1}, \ldots, B_{i-1}, C_{1}, \ldots, C_{k}, B_{i+1}, \ldots, B_{m}\right) \theta$ is derived with $\theta$ the mgu of $B_{i}$ and $P$. This step is repeated until one obtains a clause $A \leftarrow D_{1}, \ldots$, $D_{n}$ with all $D_{i}$ calls to usable predicates. This clause is added to the knowledge base as a lemma (cf. [Kedar-Cabelli 87] for an implementation in PROLOG).

\section{A problem when several examples are presented.}

When several examples are presented, each of them results in a new usable generalization. One can wonder whether the resulting set of clauses still improves the overall performance of the system (cf. [Keller 87], [Minton 88]).

Example : consider the following clauses with the PROLOG computation rule (i.e. depth-first, leftto-right) :

$\mathrm{p} \leftarrow \mathrm{q}_{1}, \ldots, \mathrm{q}_{\mathrm{n}}, \mathrm{r}_{1}, \mathrm{~s}_{1}, \ldots, \mathrm{s}_{\mathrm{m}}$

$p \leftarrow q_{1}, \ldots, q_{n}, r_{2}, s_{1}, \ldots, s_{m}$

$p \leftarrow q_{1}, \ldots, q_{n}, r_{k}, s_{1}, \ldots, s_{m}$

where $p, q_{i}, r_{h}, s_{j}$ may be arbitrary atorns in first order logic.

Suppose all these clauses have just been added to the PROLOG knowledge base. For examples only covered by the last clause, performance will be rather bad, since the subgoals $q_{1}, \ldots, q_{n}$ have to be executed $\mathrm{k}$ times. Therefore the utility of this kind of system is low, and the obtained concept description can hardly be called operational. A better performance for PROLOG would be obtained by :

$\mathrm{p} \leftarrow \mathrm{q}_{1}, \ldots, \mathrm{q}_{\mathrm{n}}, \mathrm{r}, \mathrm{s}_{1}, \ldots, \mathrm{s}_{\mathrm{m}}$

$\mathrm{r} \leftarrow \mathrm{r}_{1}$

$\mathrm{r} \leftarrow \mathrm{r}_{2}$

$\cdots$

$r \leftarrow r_{k}$

Although $\mathrm{r}$ is possibly not an operational concept, the overall problem solving behaviour of this set of rules is better than for the previous set, because backtracking is avoided. The utility of the concept definition has increased. Furthermore, $\mathrm{r}$ can be operationalized using the examples from which the rules were derived. All this must be seen in the context of operationality/generality tradeoff (cf. [Segre 87], [Braverman 88]). 
This example shows that learning may actually slow down the system (cf. [Markovitch 88], [Tambe 88], [Minton 88]) and that generalization by combining rules is useful.

\section{Generalizing learned rules.}

\subsection{A new resolution strategy.}

The idea is then to replace too specific rules by one or more general ones so that the operationality of the concept will improve. Observed from the viewpoint of corresponding proof trees, one can see this as the second generalization step of EBL : structural generalization (cf. [De Jong 86], [De Jong 88]). This generalization step will go as deep as possible, not only generalizing the structure, but also the meaning of the proof trees.

We will describe the technique as applied on two examples. We suppose two examples $E_{1}$ and $E_{2}$ are given such that applying EBL as described in section 2 yields two different clauses.

Without loss of generality, we can assume that the same clause is used at the top level of both examples ${ }^{1}$. Let $A \leftarrow B_{1}, \ldots, B_{m}$ be that clause. With $B_{i}$ a call to a non-usable concept, we can distinguish two cases :

- In both examples, the same clause $P \leftarrow C_{1}, \ldots, C_{k}$ is used to solve the instance of $B_{i}$ : the clause is then replaced by $\left(A \leftarrow B_{1}, \ldots, B_{i-1}, C_{1}, \ldots, C_{k}, B_{i+1}, \ldots, B_{m}\right) \theta$ with $\theta$ the mgu of $B_{i}$ and $P$.

- Both examples use a different clause to solve the instances of $B_{i}$. Let $B_{i} \theta_{1}$ and $B_{i} \theta_{2}$ be the two instances.

- One can find a clause $P \leftarrow C_{1}, \ldots, C_{k}$ such that there is a proof for $B_{i} \theta_{1}$ and a proof for $B_{i} \theta_{2}$ starting with that clause : replace then both subproofs by the new subproofs, so that the first case becomes applicable (to prevent infinite looping, one might put a bound on the depth of the proof trees of both examples and apply this step only if the depth bound is not exceeded).

- Otherwise $B_{i}$ is considered as pseudo-operational ; the instances $B_{i} \theta_{1}$ and $B_{i} \theta_{2}$ are used to learn two new clauses for $B_{i}$ and make $B_{i}$ operational.

This transformation is repeated until one obtains a clause $A \leftarrow D_{1}, \ldots, D_{n}$ where all calls $D_{i}$ are usable or pseudo-operational.

The algorithm is implemented in BIM-Prolog on Sun Workstations.

\subsection{Example.}

In order to give a clear example we will illustrate the technique on a small one, adapted from the hate-kill example of [Mitchell 86]. Larger examples and results can be found in [Sablon 88]. Suppose the knowledge base contains among others the clauses of fig. 1. Suppose also that the trees $t_{1}$ (fig. 2) and $t_{2}$ (fig. 3) have been derived from the examples kill(jack,john) and kill(sam,robert). Furthermore, suppose that the two derived clauses are selected to generalize.

First we apply resolution using the same clauses of both the examples. The clause in the top of both $t_{1}$ and $t_{2}$ is the same : by consequence it is also used in the resulting common proof tree (fig. 4). For the subgoals of the common clause (i.e. hate $\left(\_x, y\right)$, possess $\left(\_x, \ldots z\right)$ and weapon( $\_$) ) we do the same. For the instantiation of hate $(x, y)$ both proof trees again use the same clause, so the common proof tree uses it also. For its subgoals we have : the instantiations of married_to $\left(x_{2}, u\right)$ are leaves in $t_{1}$ and $t_{2}$, so it is a leaf of the common proof tree. For loves $(y, \ldots u)$ both proof trees use a different clause. We try to find a correspondence in meaning. Neither of the two clauses used

1) Note that if both proof trees do not use the same top level rule for $A$, we can immediately apply the second step on $\mathrm{A}$. 
(1) $\operatorname{kill}\left(\_x,-y\right) \leftarrow \operatorname{hate}(\alpha x,-y)$, possess $(x,-z)$, weapon $(z)$.

(2) bate $(x, y) \leftarrow \operatorname{married}\left(x_{,}, u\right)$, loves $(-y,-u)$.

\{ 3 \} loves $($ y, _u $) \leftarrow$ is $\left(\_u\right.$, , v $)$, likes $\_y$, w $\left.v\right)$.

(4) loves $(y,-u) \leftarrow \operatorname{seduce}(u, y)$,

(5) Ioves $(y, \ldots u) \leftarrow$ attracted_to $(y,-u)$.

(6) loves $(y, y, u) \leftarrow \operatorname{adores}(y, u)$.

(7) attracted_to $(y, \ldots u) \leftarrow \operatorname{seduce}([u,-y)$.

(8) attracted_to $(y, \ldots) \leftarrow$ charms $(-y,-u)$.

191 attracted_to $(y, \ldots u) \leftarrow i s\left(\_u, \_v\right)$, likes $\left(\_y, z v\right)$.

(10) possess $(x, \ldots z) \leftarrow$ buy $\left(\_x, z\right)$.

(11) possess $(x, z) \leftarrow \operatorname{steal}(x, z)$.

$\{12\}$ possess $(x, z) \leftarrow$ giveto $(\ldots w, \ldots x,-z)$.

\{13\} weapon $\left(\_z\right) \leftarrow \operatorname{sharp}(\ldots z)$, steel $(z)$.

(14) weapon $(z z) \leftarrow \operatorname{sharp}(z z)$, solid $(z)$.

\{15\} weapon $(z z) \leftarrow \operatorname{gun}(z)$.

(16) solid $(z) \leftarrow \operatorname{steel}\left(\_z\right)$.

(17) married_to(john, lisa).

\{ 18 \} married_to(sam,emma).

$\{20\}$ is(lisa,blond).

(21) likes(jack,blond).

(22) seduce(emma,robert).

\{23\} give_to(peter,sam,knife2).

(24) buy(jobn,knife1).

(25) sharp(knife1).

(26) sharp(knife2).

(27) solid(knife1).

(28) steel(knife2).

Figure 1. Part of a knowledge base.

for loves $(y,-u$ ) (cf. 3 and 4) in the proof trees is more general than the subtree in the other proof tree. There are more clauses for loves/2 in the knowledge base (cf. 5 and 6 ).

The first one can be used to prove both instantiations of loves $(-\mathrm{y}, \mathbf{u})$ : it is part of the common proof tree. For attracted_to $(y, \ldots u)$ there is no known clause to prove both instantiations, so it must be pseudo-operational. We cannot extend the common proof tree any further for attracted_to $\left.L_{\mathbf{y}}, \mathrm{u}\right)$. What we can do is use the two subtrees to operationalize attracted_to/2. In this case the subtrees are only of depth two, so that this operationalization does not yield any new clause.

Next atom is possess $(x, z)$. Since there is no correspondence in meaning between $t_{1}$ and $t_{2}$, it is a leaf in the common proof tree. The corresponding subtree of $t_{1}$ is used to operationalize its root.

For the third atom weapon $(z)$ a different clause is used in both proof trees. But the clause of $t_{1}$ can be used in $t_{2}$ too, so it is in the common proof tree. $\operatorname{Sharp}(z)$ and $\operatorname{solid}(z)$ are both leaves of $t_{1}$, so the common proof tree comes to an end. 


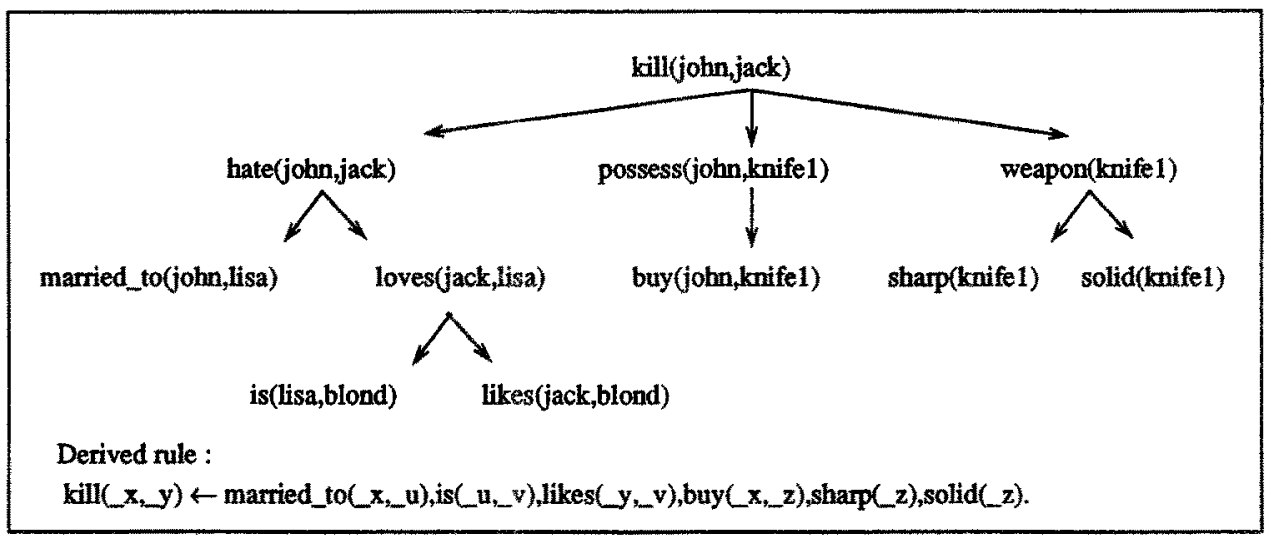

Figure 2. Proof Tree $t_{1}$ of the First Selected Example.

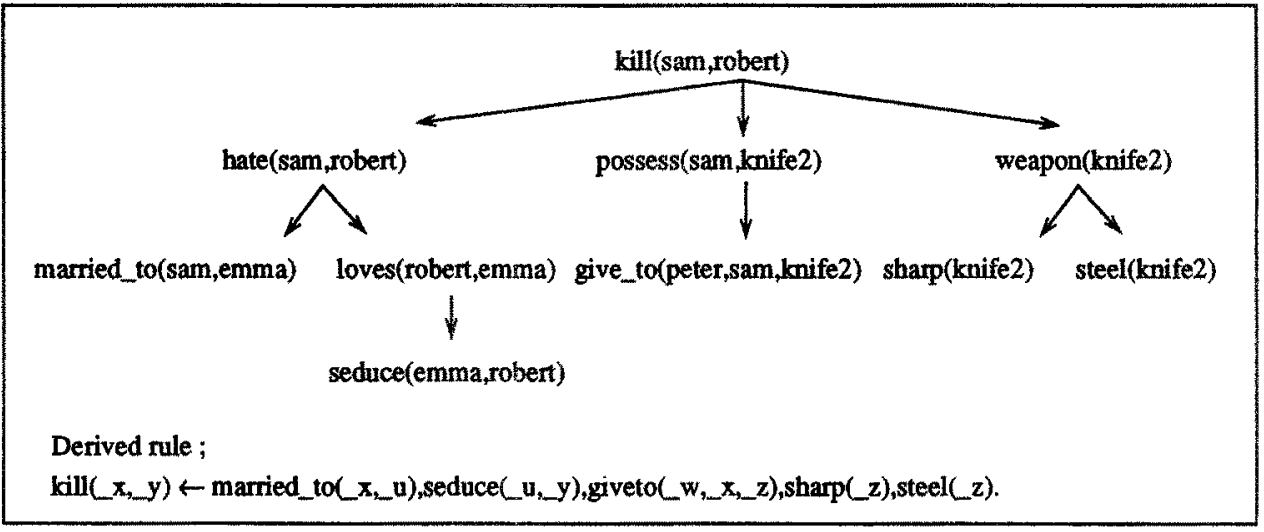

Figure 3. Proof Tree $t_{2}$ of the Second Selected Example.

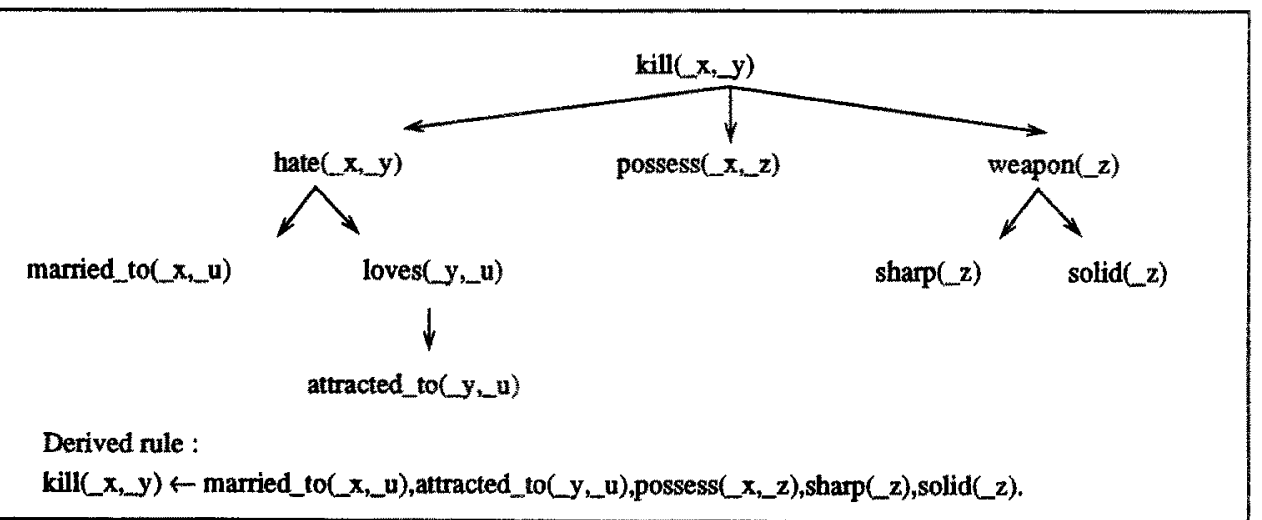

Figure 4. Generalized clause and corresponding proof tree. 


\section{Discussion.}

The desired equilibrium between generality and usability depends on the definition of a conceptual distance (cf. [Michalski 83]) between different examples (or, alternatively, between the corresponding proof trees). The distance allows one to select the examples whose rules should be combined. The larger the distance between examples to be generalized is allowed, the more general and less usable, but the more useful the new rule will be. Consequently, the conceptual distance is highly dependent upon the desired utility of the concept definition. Combining usability and utility we can optimize the operationality.

Each time a new example is presented, adding its corresponding rule can decrease operationality, because certain objectives with respect to utility are no longer reached. As mentioned above, this can happen when a concept description becomes too redundant. Therefore generalization should be considered (using the conceptual distance) each time a new example is presented. In general, one could apply techniques as in [Keller 87] or [Minton 88] to detect the need for generalization.

The purpose of the second step of the algorithm in section 4.1 is to identify possible common parts of proof trees with respect to their meaning. Especially when certain (sub)problems have several possible proof trees, it is important to recognize possible common parts. Structurally generalizing without taking into account their meaning, may lead to overgeneralized rules. The particular situation of having several possible proofs occurs in each EBL system after learning new rules. So it is very useful to perform this step in EBL systems.

\section{Related Work.}

The roots of our EBL method lie in the work of [Mitchell 86], [De Jong 86] together with others as [Hirsch 87] and [Kedar-Cabelli 87]. Our notions of operationality (usability and utility) are based on the work of [Keller 87] and [Minton 88].

In [Braverman 88] boundaries of operationality are searched We extend this notion to the new definition of operationality by searching boundaries of generality. The advantage of our method is that the derived rules are more general, therefore more useful and more operational since we also operationalize the subgoals on the boundary. Another difference is that their search for a boundary of operationality is based upon (the generalized proof tree of) only one example, while in our search several examples are used. This increases the probability that the new rule will be more useful.

According to [Mitchell 86], our method is also related to the one of [Kedar-Cabelli 84] and [KedarCabelli 85]. Their technique also recognizes common parts of proof trees, but uses analogy on the differing portions. The difference between their approach and ours is that our system also compares the meaning of subtrees which use different rules. Comparing proof trees uses domain knowledge, so that new deduced knowledge is still correct.

Furthermore techniques as generalizing number (cf. [Shavlik 87a], [Shavlik 87b] and [Cohen 88]) are related to generalizing from rules that use recursive predicates. [Cohen 88] also applies his method on generalizing from multiple examples. However, he concentrates on the structure of the proof trees with respect to repeated rule applications, while we concentrate on the meaning. Hence, we believe that the techniques are complementary.

\section{Conclusion.}

We have developed a technique for EBL in the case of multiple examples. A new derived rule is not just added to the knowledge base when the operationality criterion is no longer reached. Instead this rule is generalized, together with existing rules, in order to operationalize the concept description. Furthermore the subconcepts are operationalized. In this way we achieve an equilibrium in the trade-off between generality and operationality. 


\section{REFERENCES.}

Braverman, M.S., Russell, S.J., Boundaries of Operationality, Proceedings of the fifth International Conference on Machine Learning, Ann Arbor, MI, Morgan Kaufmann, 1988, pp. 221-234.

Cohen, W.W., Generalizing Number and Learning from Multiple Examples in Explanation Based Leaming, Proceedings of the fifth International Conference on Machine Learning, Ann Arbor, MI, Morgan Kaufmann, 1988, pp. 256-269.

De Jong, G.F. and Mooney, R.J., Explanation Based Leaming : An Altemative View. Machine Learning 1, 2 (April 1986), pp. 145-176.

De Jong, G. , Some thougths on the Present and Future of Explanation-Based Learning, Froceedings of the 8th ECAI, Pitman, London, 1988, pp. 690-697.

Genesereth, M.R., Nilsson, N.J., Logical Foundations of Artificial Intelligence. Morgan Kaufmann, Los Altos, 1987.

Hirsch, H., Explanation Based Generalization in a Logic-Programming Environment. IJCAI 87. Proceedings of the tenth International Joint Conference on Artificial Intelligence, 1987, pp. 221-227.

Kedar-Cabelli, S.T., Analogy with purpose in legal reasoning from precedents, (Technical Report LRP-TR-17), Laboratory for Computer Science Research, Rutgers University, New Brunswick, NJ, 1984.

Kedar-Cabelli, S.T., Purpose-directed analogy, Proceedings of the Cognitive Science Society Conference, Irvine, CA: Morgan Kaufmann, 1985.

Keller, M., Defining Operationality for Explanation-Based Learning, Proceedings of the Sixth National Conference on Artificial Intelligence, 1987, pp. 482-487.

Kowalski, R.,Logic for problem solving, North Holland, New York, 1979.

Markovitch, S., Scott, P.D., The Role of Forgetting in Leaming, Proceedings of the fifth International Conference on Machine Learning, Ann Arbor, MI, Morgan Kaufmann, 1988, pp. 459465 .

Minton, S., Carbonell, J.G., Strategies for Learning Search Control Rules : An Explanation Based Approach, IJCAI 87. Proceedings of the tenth International Joint Conference on Artificial Intelligence, 1987, pp. 228-235.

Minton, S., Quantitative Results Concerning the Utility of Explanation-Based Learning. Proceedings of AAAI 88, 1988, pp. 564-569.

Michalski, R.S., Leaming From Observation : Conceptual Clustering, Machine Learning, an Artificial Intelligence Approach, Springer-Verlag, 1983, pp. 331-364.

Mitchell, T.M., Keller, R. and Kedar-Cabelli, S., Explanation Based Generalization. A Unifying View. Machine Learning 1, 1 (January 1986), pp. 47-80.

Sablon, G., Automatisch leren op basis van verklaringen voor trainingsvoorbeelden, Licentiate's thesis, Dept. of Computer Science K.U.Leuven, june 1988 (in Dutch).

Segre, A.M., On the operationality/generality trade-off in explanation-based leaming. IJCAI 87. Proceedings of the tenth International Joint Conference on Artificial Intelligence, 1987, pp. 242-248.

Shavlik, J., De Jong, G., BAGGER : an EBL system that extends and generalizes explanations. Proceedings of the Sixth National Conference on Artificial Intelligence, 1987, pp. 516-520.

Shavlik, J., De Jong, G., An explanation-based approach to generalizing number. IJCAI 87. Proceedings of the tenth International Joint Conference on Artificial Intelligence, 1987, pp. 236-238.

Tambe, M., Newell, A., Some Chunks Are Expensive Proceedings of the fifth International Conference on Machine Learning, Ann Arbor, MI, Morgan Kaufmann, 1988, pp. 451-458. 